\title{
Los talleres de tesis como aproximación a una comunidad de práctica ${ }^{1}$
}

\section{Thesis workshops as approaches towards a community of practice}

ISSN 1510-2432 - ISSN 1688-9304 (en línea) - DOI: https://doi.org/10.18861/cied.2017.8.1.2637

Lorena Fernández Fastuca

Doctora (Cand.) en Educación, Universidad de San Andrés, beca doctoral del Consejo Nacional de Investigaciones Científicas y Tecnológicas (CONICET). Licenciada en Ciencias de la Educación, Universidad Católica Argentina (UCA). Profesora, departamento de Educación, UCA. Especialista en didáctica nivel postgrado con énfasis en la formación de investigadores.

Jennifer Guevara

Doctora (Cand.) en Educación, Universidad de San Andrés, beca doctoral del Consejo Nacional de Investigaciones Científicas y Tecnológicas (CONICET). Licenciada en Ciencias, Universidad de San Andrés. Ex docente en Facultad Lationoamericana de Ciencias Sociales (FLACSO) y Universidad de San Andrés.

Fecha de recibido: 13/05/2016

Fecha de aceptado: 20/04/2017

\section{Resumen:}

Uno de los desafíos de la formación de investigadores durante los estudios de posgrado es formar en el quehacer de la investigación que involucra no solamente el desarrollo de competencias sino también la inmersión en una comunidad de práctica: la comunidad académica. Los talleres constituyen espacios en los que se establece una interrelación entre teoría y práctica que favorece el aprendizaje del quehacer de la investigación. Este artículo explora los talleres de tesis en una universidad argentina como espacios de aproximación a una comunidad de práctica. Propone un abordaje centrado en los dispositivos pedagógicos, el que puede resultar de utilidad para la investigación pedagógico-didáctica sobre la formación de posgrado. Se observaron los encuentros de dos talleres de un mismo programa doctoral en ciencias sociales y se realizó un análisis temático. Se encontró que los talleres proponen tareas significativas para la comunidad académica, proveen un ambiente controlado pero no simulado de aprendizaje y favorecen el aprendizaje del repertorio y el intercambio con pares y docentes. Empero, cuando la evaluación es preponderante, el taller propicia lógicas de aprobación más que de aprendizaje. En definitiva, el marco de comunidades de práctica es propicio para el abordaje de la transmisión de saberes prácticos en el marco de la educación de posgrado al no enfocarse solamente en lo que sucede en la institución educativa y tomar al aprendizaje como un proceso que se desarrolla en tanto se participa en una comunidad de práctica.

Palabras claves: talleres de tesis, educación doctoral, formación de investigadores, comunidades de práctica, participación periférica legítima

\begin{abstract}
:
One of the challenges of research training in postgraduate studies is to train early stage researchers in this area. Such training not only involves the development of skills but also the immersion in a community of practice; the academic community. Workshops are spaces where interplay between theory and practice promoting learning research work is established. This article explores thesis workshops in Argentina as approaches
\end{abstract}


towards a community of practice. It focuses on pedagogic devices, which can be useful for postgraduate training pedagogical research. Two workshops of the same doctoral program in Social Sciences were observed, and the resulting data were analysed thematically. It was found that workshops offer significant tasks for the academic community, provide a controlled yet not simulated learning environment, facilitate the learning of the community repertoire and favour the exchange with peers and teachers. However, when assessment prevails the workshop focuses on obtaining passing grades rather than on learning. In short, the framework of communities of practice is significant in order to address the transmission of practical knowledge in the context of postgraduate education, since it does not only focus on the institutional process but also conceives learning as a process that develops alongside participation in a community of practice.

Keywords: thesis workshops, doctoral education, research training, communities of practice, peripheral legitimate participation

\section{Introducción}

Uno de los principales espacios de formación de los investigadores en distintos países son las universidades, concretamente en los doctorados (Clark, 1995). Esta formación implica la inserción en una comunidad de práctica: la comunidad académica. Así, la educación doctoral enfrenta el desafío de formar a sus estudiantes para una práctica profesional específica dotándolos de herramientas para afrontar su complejidad. Durante su formación los investigadores deben enfrentarse a la definición de un objeto de estudio, la conducción de una investigación y la publicación de artículos entre otras tareas propias de un investigador, y que difícilmente han realizado antes. En las ciencias sociales en particular el taller de tesis es una práctica dirigida a la orientación en dichas actividades en función de la producción del proyecto de investigación. Sin embargo, es poco lo que se conoce sobre dichos talleres hasta el momento. En este artículo nos proponemos explorar los talleres de tesis en la formación de investigadores en la Argentina como espacios de aproximación a una comunidad de práctica y a su tarea.

Las comunidades de práctica son definidas como un "grupo de personas interdependientes socialmente que comparten objetivos y prácticas mutuamente definidos" (Barab, Barnett y Squire, 2002:6), es decir, una comunidad que se reúne en torno a una práctica profesional. Los académicos de una misma disciplina pueden ser considerados miembros de una comunidad de práctica ya que comparten una tarea, manejan un sistema de normas y valores comunes y circulan por el mismo tipo de instituciones. Insertarse en una comunidad implica familiarizarse con su tarea, adquirir su sistema de normas y valores y aprender a circular por sus instituciones. Las comunidades tienen sistemas previstos para la inclusión de nuevos miembros, y el doctorado se constituye en el mecanismo de inserción de la comunidad académica. En particular, los talleres de tesis nos permiten observar una fase de este proceso, aquella que se focaliza en la práctica de los saberes del oficio del investigador.

Los talleres tienen ya algunas décadas de introducción en el ámbito educativo aunque sus orígenes se remontan al modo de organización de los gremios medievales cuya característica distintiva era el reunirse alrededor de un hacer. De allí emerge el taller como dispositivo pedagógico, un espacio de articulación entre teoría y práctica.

Para alcanzar nuestro objetivo presentaremos, en primer lugar, algunas referencias conceptuales vinculadas al taller como dispositivo pedagógico y a las comunidades de práctica. En segundo lugar presentaremos el diseño metodológico que ha guiado este trabajo. Finalmente, expondremos nuestros resultados con vistas a dilucidar en qué medida los talleres observados se constituyen en espacios de inserción en una comunidad y de aprendizaje de la práctica investigativa. 


\section{Antecedentes y fundamentación teórica El taller}

Existe una larga tradición que concibe a la investigación como un oficio (Bourdieu, Chamboredon y Passeron 2008; Wainerman, 2011; Wright Mills, 1959) por lo que no es extraño remontarnos a los gremios medievales para pensar los talleres de tesis.

Entre los siglos XIII y XIV el aprendizaje de los oficios se realizaba en el taller del maestro artesano. Este proceso, que solía durar varios años, involucraba al maestro -dueño del taller y poseedor del saber del oficio- y a uno o dos aprendices. La finalización de la formación estaba marcada por la realización de una obra maestra que permitía al aprendiz ascender a la posición de maestro (Baruque, 2001; Menjot, 2010). La relación entre el maestro artesano y su aprendiz estaba mediada por la obra propia de su oficio. Así, el aprendizaje del oficio ocurría de la mano de un experto y en el proceso mismo de producción.

En el mismo período emergieron otros modos de transmisión del saber elaborado tales como las universidades cuya labor se asociaba, principalmente, a la reflexión teórica. Estas instituciones se centraban en las artes liberales. La Edad Media se caracterizó por diferenciar los espacios de transmisión del saber elaborado: en las universidades se dio cabida a los saberes intelectuales y en los gremios a los saberes vinculados a lo manual. Asimismo, los primeros gozaban de mayor jerarquía.

En las últimas décadas del siglo XX la importancia de los conocimientos prácticos ha sido recuperada por distintos representantes de las ciencias sociales, entre los que puede citarse a Atkinson y Claxton (2000), Bourdieu, Chamboredon y Passeron (2008), Schön (1992) y Sennett (2009). Estos saberes están presentes en campos tan diversos como el de un docente, un enfermero o un investigador. Son saberes que se aprenden de la mano de un miembro de una comunidad (que podemos identificar con el maestro artesano) y en interacción con otros de sus miembros más o menos experimentados que también participan del proceso de aprendizaje (Lave, 2011).

En la actualidad, la modalidad taller se ha abierto camino en las instituciones educativas del nivel universitario. Algunos de sus elementos han sido recuperados y reinterpretados por la didáctica y transformados en un dispositivo pedagógico. En este sentido, repensar la formación en los saberes de la práctica en la educación de posgrado a través de los talleres de tesis podría implicar reimaginar, en alguna medida, la lógica de trabajo de los gremios medievales. La complejidad inherente a la práctica actual requiere ofrecer modos de formarse distintos de los brindados tradicionalmente por la educación. En particular, dispositivos pedagógico-didácticos que privilegien un acercamiento a la práctica sin distanciarse de la teoría.

En lo que respecta a la formación de investigadores muchos programas doctorales en ciencias sociales contemplan la realización de talleres (también llamados seminarios) orientados a guiar en la realización de la tesis. Entre ellos se encuentran dispositivos de distinto grado de sistematicidad desde los Brown Bag Seminars ${ }^{2}$ hasta los talleres de tesis y los de escritura.

Los talleres de tesis han sido poco estudiados y creemos que esto se debe a que existen en los países latinoamericanos principalmente. En los países angloparlantes existirían principalmente seminarios de investigación y grupos de escritura. Contrariamente, la literatura se ha concentrado en los talleres de escritura (Colombo, 2012; Boud y Lee, 
2005; Carlino, 2012; Carrasco, 2011), que se diferencian de los de tesis pero parece haber poca claridad respecto a los objetivos que persiguen uno y otro y en qué consiste cada uno. La confusión entre ambos es evidente para algunos especialistas:

“...hemos podido observar que, bajo esta denominación [taller de tesis], existen muy diversos contenidos objeto de enseñanza y que no necesariamente estos talleres abordan la cuestión de la escritura. Algunos se encaran como seminarios de metodología, otros se abocan a mostrar investigaciones hechas por miembros de los equipos docentes, etc." (Arnoux, 2004:5).

Según Arnoux (2004) es necesario que un taller de tesis aborde cuestiones tanto de metodología de la investigación como de la escritura de la tesis. No habría tesis como texto sin el trabajo de investigación previo. En este artículo, como se ha indicado, interesan particularmente los talleres de tesis ya que son los que se encargan específicamente del desarrollo de los saberes del quehacer de la investigación, no solo de su escritura. Asimismo, al haber sido poco indagados hasta el momento, permite aportar a la reflexión pedagógico-didáctica sobre el tema.

Dentro de los estudios sobre los talleres de tesis no hay acuerdo entre si son espacios en los que se aprende a hacer investigación investigando al modo de los talleres de artesanos (Wainerman, 2011) o si incluyen también la enseñanza de conocimientos codificados de metodología de la investigación (Difabio y Heredia, 2013). Lo que parece claro es que entienden a la tesis más allá del texto escrito como una investigación y a esta como un proceso durante el cual se requiere orientación en sus distintas etapas.

El taller es, asimismo, un dispositivo pedagógico en el que se establece una relación inseparable entre teoría y práctica asociada a un posicionamiento particular del docente frente al aprendizaje, el saber y la enseñanza (Faiver, McNally y Nims, 2000; Jones, 2000; Lopes Espinoza y Weiss, 2007). Se trata de un aprender haciendo guiado por la reflexión sobre el hacer. Desde esta concepción el taller alcanza toda su potencialidad cuando el aula se convierte en un espacio habilitado para indagar, intercambiar ideas y equivocarse (Pasel y Asborno, 1991; De Vita, 2012). El docente incentiva y orienta a los alumnos en su proceso de aprendizaje (Pasel y Asborno, 1991). El taller admite la puesta en juego de una amplia variedad de métodos didácticos en pos de dar lugar a la relación teoría-práctica deseada. Desde esta perspectiva, la propuesta del taller se funda en una concepción de aprendizaje que resalta su carácter de proceso continuo y de ampliación permanente de experiencias previas y trabaja a partir de las dudas, los errores y la necesidad del sujeto que aprende. Asimismo promueve el contacto con el medio y la interacción con los otros (Pasel y Asborno, 1991). En definitiva, los talleres de tesis pueden ser ocasiones de aproximación a la práctica del quehacer de la investigación ${ }^{3}$, permiten a los alumnos aprender en la misma ejecución de una tarea propia de la comunidad académica como comunidad de práctica. En este sentido, el taller puede colaborar con el proceso de inmersión en una comunidad de práctica y ofrecer oportunidades de aprendizaje como participación, tal como veremos en el próximo apartado.

\section{Las comunidades de práctica}

Décadas atrás Schön identificó el tipo de saber "que los prácticos muestran algunas veces en situaciones de la práctica que resultan singulares, inciertas y conflictivas" (1992: 33) y lo denominó conocimiento práctico. Para el autor, cuando alguien aprende una práctica 
esta se inicia en las tradiciones de una comunidad de prácticos y del mundo particular que estos habitan. En este proceso aprende sus convenciones, limitaciones, lenguajes y sistemas de valoración, sus repertorios de ejemplos, su conocimiento sistemático y sus patrones de conocimiento en la acción (Ibíd.: 45). Así, Schön identifica al taller como un espacio privilegiado para el aprendizaje de una práctica en un contexto que se aproxima -y a la vez controla- el mundo de la práctica.

En línea con las ideas de Schön, Wenger (1998) propone una conceptualización de las comunidades de práctica e identifica tres características centrales: (1) empresa común; (2) compromiso mutuo y (3) repertorio compartido. La empresa común de una comunidad es la tarea que los reúne, que es definida a través de procesos de negociación entre sus miembros insertos en contextos históricos, culturales e institucionales específicos. Se trata de un proceso continuo y de larga duración en el que se negocian tanto la definición de la empresa común de la comunidad como el modo de participación de sus miembros. Esta participación se caracteriza por el compromiso mutuo, que no está dado simplemente por relaciones sociales cercanas o por proximidad geográfica sino por la reunión en torno a la tarea de la comunidad. Finalmente, los miembros de una comunidad de práctica comparten un repertorio -marca de identidad compartida- que los diferencia de otras comunidades. Dicho repertorio incluye palabras, símbolos, historias, modos de hacer y herramientas, entre otros. Las comunidades de práctica comparten modos de ver el mundo y de actuar e interactuar en él. Si bien el concepto de comunidad de práctica habilita un análisis a distintos niveles y dimensiones, en este trabajo nos ocuparemos de aquellas que se conforman alrededor de una práctica profesional.

Lave y Wenger (1991) asocian el aprendizaje a la participación social en comunidades de práctica, es decir, a lo que ocurre en la vida cotidiana en relación con las personas y con el mundo. Desde esta perspectiva Lave y Wenger argumentan que la escuela no es el único espacio para el aprendizaje y, más aún, que el aprendizaje escolar tiene ciertas particularidades que no resultan útiles para pensar el aprendizaje en otras situaciones sociales. Así, el aprendizaje deja de estar necesariamente atado a formatos institucionales y a la enseñanza escolar.

Fuller (2007) identifica cuatro puntos de ruptura en relación con la concepción de aprendizaje que plantea el marco de comunidades de práctica:

1. Pasaje de una unidad de análisis individual a una colectiva. En este proceso lo importante es la relación y no ya el individuo.

2. Posicionamiento en el centro de la escena de la noción de práctica social, en el sentido de que las personas aprenden a partir de su participación en prácticas compartidas de una comunidad que habita en el mundo.

3. Lo aprendido es indisoluble de la formación de identidad.

4. El novato no es un receptor pasivo de conocimiento sino un participante de la actividad que se encamina hacia grados más plenos de participación.

En líneas generales, se trata de recuperar que las personas, su actividad y el mundo en el que actúan e interactúan no pueden concebirse de manera separada. Esta perspectiva nos permite ampliar la mirada y reconocer instancias formativas que no necesariamente se ajustan al modelo de enseñanza y aprendizaje tradicional organizado alrededor del dispositivo de clase. 
Las comunidades tienen, además, ciclos de reproducción. De hecho, muchas de las comunidades contemplan modos de inserción de sus potenciales miembros. Los miembros plenos inscriben a los nuevos a través de la participación en actividades compartidas y centrales para la empresa de la comunidad. Desde este enfoque se aprende en compañía de otros miembros más antiguos y participando de manera periférica en tareas significativas para la propia comunidad. Por eso Lave y Wenger (1991) hablan de aprendizaje como participación, que se caracteriza por ser periférico y legítimo en tanto modo de conceptualizar la inmersión progresiva en la comunidad de práctica. La participación se diferencia de la colaboración por involucrar relaciones de distintos tipos, sean estas conflictivas o armoniosas. Asimismo, lo aprendido es indisoluble de la formación de identidad (Perry, 2004). Quien se inicia en una comunidad de práctica forja una identidad como miembro de dicha comunidad.

Ya establecidos los referentes teóricos principales que orientaron este trabajo nos preguntamos: ¿Qué posibilidades brindan los talleres de tesis para pensar el aprendizaje de los saberes de la práctica investigativa? Nuestro trabajo de investigación buscó dar una respuesta a este interrogante.

\section{Diseño y metodología}

La evidencia empírica que sustenta este artículo surge de una investigación doctoral de orientación cualitativa centrada en la formación de investigadores ${ }^{4}$. Se realizaron 60 horas de observación en dos espacios curriculares obligatorios organizados bajo la modalidad de taller en el marco de un mismo programa doctoral en ciencias sociales de una universidad de gestión estatal de la ciudad de Buenos Aires (Argentina). Dicha universidad fue seleccionada por su vasta trayectoria y por ofrecer uno de los programas doctorales más extensos del país. Es un programa personalizado en el que los doctorandos deben obtener 20 créditos en seminarios a su elección (los talleres de tesis son los únicos obligatorios) y la tesis en un plazo estipulado en seis años.

En este marco se ofrecen talleres de tesis en dos momentos: el taller I al inicio del doctorado, cuyo objetivo es orientar a los estudiantes en la elaboración de un proyecto de investigación ${ }^{5}$ y el taller de tesis II hacia la finalización del doctorado, que busca guiar en la elaboración de un plan de tesis ${ }^{6}$. El criterio principal para la selección de los talleres fue teórico: uno de cada instancia para analizar la totalidad del proceso formativo como investigadores. La selección de los cursos concretos fue guiada por razones de conveniencia: facilidad de acceso y ser dictados en el semestre en que se realizó el estudio. Los talleres tuvieron una duración de 10 encuentros de tres horas cada uno. Todos los alumnos del doctorado deben realizar, al menos, uno de los talleres mencionados. Que el alumno deba realizar uno o ambos se define en la entrevista de admisión según los antecedentes del postulante. El taller de tesis I observado estuvo a cargo de un docente-investigador y contó con 11 alumnos. El taller II estuvo a cargo de dos docentes-investigadores y al mismo asistieron 16 alumnos. En ambos casos se realizó un seguimiento de la totalidad del desarrollo del taller. Se tomaron notas de campo (toma de notas en el momento de la observación e inmediatamente posterior a ella) y se grabaron archivos de audio de cada uno de los encuentros. Posteriormente las transcripciones de dichos archivos de audio se articularon con las notas de campo. Asimismo se realizaron entrevistas informales con los docentes y algunos alumnos. La información recolectada se procesó a través de un análisis temático utilizando como herramienta el software Atlas.ti. Los nudos temáticos identificados fueron los siguientes: la tarea, el carácter 
controlado pero no simulado de la práctica en el taller, el repertorio, la importancia de las interacciones en el proceso de aprendizaje -tanto con los miembros como entre pares-, el taller como hito de ingreso a la comunidad y el posicionamiento de los alumnos y docentes frente al conocimiento. A partir de ellos se realizó una codificación selectiva.

\section{Resultados y discusión El taller: potencialidades para la inserción en una comunidad de práctica}

Los talleres de tesis observados utilizaron un método didáctico similar entre sí. En ambos casos los doctorandos debieron realizar guías de trabajo semanales que los guiaron gradualmente a la realización del proyecto de investigación o del plan de tesis, según correspondiera. Estas guías orientan a los alumnos en el avance de sus tesis brindándoles consignas que implican la reflexión y puesta en texto de distintas dimensiones de la tesis (Arnoux, 2009). Por ejemplo, una de las consignas del taller I era identificar los problemas del área temática elegida para la tesis para, a continuación, ver en cuál de ellos se inserta la tesis. En este marco general el taller de tesis I ordenaba sus encuentros a partir de una exposición teórica en la que se abordaban los contenidos del programa, y el docente evaluaba oralmente las guías de trabajo dando lugar en ocasiones a la intervención de los alumnos. Esta misma estrategia de enseñanza (guías de trabajo semanales) se resolvía de modo distinto en el taller II. Los doctorandos debían entregarlos vía correo electrónico unos días antes de cada clase y presentarlos oralmente en los encuentros durante los cuales se discutían con el resto del grupo.

A pesar de sus diferencias los talleres comparten el objetivo de acompañar a los doctorandos en el desarrollo de la tarea propia de su comunidad de práctica. A partir de la estrategia presentada cada semana los estudiantes avanzaban en la realización de su proyecto o plan de tesis. Para ello recibían orientaciones del docente a cargo del taller (también un investigador), y de sus pares en el caso del taller II. Este foco en una tarea propia y central de la comunidad de práctica diferencia a estos espacios de otros que predominan en la educación de posgrado tales como las asignaturas y los seminarios. Los docentes de los talleres de tesis inclusive manifestaron que el objetivo de estos "no es enseñar metodología de la investigación" (para ello existen los cursos de metodología) sino constituirse en un espacio donde los estudiantes puedan desarrollar una fase de su investigación. De tal modo los talleres podrían estar funcionando como un puente entre estas instituciones y la comunidad de práctica de referencia (Goodwin, 2007): la comunidad académica.

No obstante, la tarea de la comunidad no aparece representada en su totalidad en los talleres observados. Por el contrario, la labor se centró en el desarrollo de una etapa de la investigación. La recolección de datos, su análisis y la redacción del informe final no fueron abordados. A pesar de lo limitante que pueda parecer, esto en realidad permite que los alumnos se concentren en el dominio de saberes específicos del quehacer de la investigación. Si los talleres hubieran pretendido abarcar la totalidad del proceso investigativo habría sido en detrimento de la profundidad en el abordaje de los saberes. De este modo, los talleres observados promovieron un acercamiento a la práctica profesional real en un contexto controlado. En ellos, la posibilidad de fracaso está controlada, atenuada por las revisiones y orientaciones que recibe el doctorando. El siguiente fragmento ${ }^{7}$ ejemplifica el ensayo en la definición de objetivos orientada por los docentes:

“Clara: A ver, se me ocurre una cosa pero no sé si está bien. ¿Puedo?

Mauro: Sí. 
Clara: Mirá, a ver si está bien como objetivo: 'Explorar acerca de los efectos sociodemográficos que la evolución y composición de la masa societaria de los clubes seleccionados tuvieron para las zonas donde se ubicaron'.

Mónica: Digamos, es correcto. Ahora habría que ver si es teóricamente consistente. Si es posible pensar desde el punto de vista teórico que la instalación de un club, de una organización, de lo que fuera puede tener impactos sociodemográficos.

Clara: Claro". (Fragmento observación taller de tesis II)

La retroalimentación inmediata que reciben los doctorandos en los talleres basados en este método didáctico favorece su avance a partir de los comentarios recibidos. De acuerdo con Kiley (1996) este tipo de retroalimentación es esencial para el trabajo y bienestar de los alumnos que se están embarcando en un período de intenso trabajo en un solo proyecto. Este contexto controlado es de gran importancia ya que en ciencias sociales sigue prevaleciendo el trabajo individual por sobre la conformación de equipos y el comienzo en la investigación ocurre en soledad con la tesis doctoral (a excepción del director de tesis), por lo que el aprendizaje se realiza con actividades de alta significación para el tesista. Esto puede significar una relación más emotiva con la tarea en la que el fracaso tendría un costo afectivo mayor. Por este motivo, el contexto controlado que ofrecen los talleres puede atenuar dicho costo así como el cognitivo. De ahí su importancia como instancia formativa y de acompañamiento a los doctorandos en el inicio formal de su carrera como investigadores. La participación en estas prácticas promueve la apropiación de la cultura así como también favorece los procesos de reflexión y adquisición del conocimiento. En palabras de Litwin: "Si los estudiantes participan efectivamente en la organización y desarrollo de una situación [...] los aprendizajes son más duraderos, impactan en sus conciencias, promueven reflexiones y permiten mejores procesos de autoevaluación". (2008:102).

Para colaborar en la resolución de las guías y acortar la distancia entre el espacio del aula y la práctica de la investigación los docentes usualmente transmitieron su experiencia personal para aconsejar a los alumnos o ejemplificar la toma de decisiones en el proceso de construcción de la tesis. Los siguientes fragmentos son ejemplos de esto:

“Mónica: Todo lo que leí de [esa línea de investigación] me sirvió para poner un párrafo, tengo hasta visualizado el lugar de la tesis donde lo puse para decir 'este problema se aborda en la bibliografía internacional desde dos perspectivas. Los referentes de la perspectiva tal son estos. Y yo lo voy a abordar desde esta'. Solo un párrafo". (Fragmento observación taller de tesis II).

“Ezequiel: Una pregunta, esto que planteabas en tu ejemplo: ‘no me voy a pelear con los existencialistas, en esta introducción' y que planteaste una definición en la que se plasma el problema desde el punto de vista esencialista.

Bernardo: Yo empecé por ahí. El principal cruce o el principal debate entre las distintas líneas de investigación, los distintos cruces de investigación que hay adentro de esta problemática son los que se dan entre los esencialistasy, llamémosles, constructivistas...". (Fragmento observación taller de tesis I)

En los talleres el foco estaba puesto tanto en la tarea como en la reflexión sobre la misma. En el taller de tesis II, luego de la presentación del trabajo de los estudiantes cada uno discutía sobre cómo se había resuelto la actividad y los desafíos que habían enfrentado. El aprender haciendo que propicia el taller no estaría completo si no existiera reflexión sobre lo que se hace, cómo se lo hace y por qué se lo hace (Schön, 1992). Así, teoría y 
práctica se conciben de manera integrada (Barros, 1977; Buysse, Sparkman y Wesley, 2003). De esta forma el puente entre la educación de posgrado y la práctica profesional se construye a partir de la integración entre teoría y práctica y del papel activo que tienen los doctorandos en los talleres de tesis.

En primer lugar, el principal objetivo de estos cursos es que los alumnos trabajen sobre sus propios proyectos de tesis a partir de las guías que les permiten realizar sucesivas reescrituras. En concordancia con lo expuesto por Vitale (2009), la posibilidad de volver sobre lo escrito y discutirlo con otros lleva a una progresiva apropiación de un saber disciplinario e incide en la reconfiguración de la subjetividad del doctorando, de consumidor de conocimientos a productor de ellos dentro de un campo disciplinar específico (Lovitts, 2005).

Además del aprendizaje de la tarea específica se observó que en los talleres se aprende el repertorio de la comunidad (Wenger, 1998). En los talleres de tesis observados los alumnos profundizaron sus conocimientos sobre las herramientas de la comunidad (proyecto de tesis, la tesis, las técnicas de investigación), adoptaron parte de su lenguaje (el discurso científico) y vivenciaron o identificaron algunos de sus roles (jurado de tesis, comentaristas en los congresos, par evaluador de una revista científica). En cuanto al lenguaje académico en algunas ocasiones los docentes piden rigurosidad y precisión al hablar:

“Mónica: ¿Urbano también es una categoría nativa?

Paula: No, esto no. Yo no sabía cómo ponerlo porque tampoco hay... o hay sectores de clase bastante alta que no viven en el territorio pero que construyen countries...

Mónica: Si vas a usar categorías nativas usá todo categorías nativas". (Fragmento observación taller de tesis II)

Estos pedidos de mayor precisión son estrategias que modelan paulatina y progresivamente el discurso académico característico de la comunidad a la que se insertan, discurso que cumple con los requisitos científicos. Así modelan el trabajo académico y son ejemplos del desarrollo, de la ejecución de la actividad. Asimismo, en este proceso de aprendizaje del saber hacer caracterizado por la participación se observó cómo tenía lugar la conformación de una identidad: saber hacer e identidad definen al participante pleno de la comunidad. En este sentido no se trata solamente de adquirir habilidades o conocimientos sino actuar en formas que sean reconocidas y valoradas por otros miembros (Contu y Willmott, 2003). Es decir, no es aprender a llevar a cabo una investigación sino formarse como investigador. En este proceso de inmersión los novatos, en tanto participantes periféricos, construyen una identidad negociando significados con la comunidad en un proceso que habilita tanto la reproducción de la comunidad como su transformación.

En esta línea se observó que los talleres promovían las interacciones entre miembros de distinto nivel de participación (además de novatos y experimentados puede haber miembros con un grado de inserción intermedio que colaboren en el proceso). La primera relación que podemos identificar es la que se establece entre los miembros plenos y los novatos. En los talleres de tesis los alumnos, en lugar de hacer su proyecto o plan de investigación en una relación diádica con su director de tesis contaron, además, con las orientaciones del profesor del taller antes de entregarlo para su evaluación al programa doctoral. 
En segundo lugar, los talleres observados estuvieron pensados con el fin de incrementar las interacciones entre los pares. En ambos casos se evidenciaron instancias de discusión grupal sobre los obstáculos encontrados en la ejecución de la propia tarea. Por ejemplo, la consigna semanal era puesta en discusión con el resto del grupo. El siguiente fragmento muestra modos de interacción entre pares:

"Lucrecia: Yo me imagino un capítulo que sería vincular informalidad urbana y procesos migratorios en la ciudad de Buenos Aires, eso como un panorama teórico y datos, ¿no? Susana: ¿No sería más trayectorias residenciales e informalidad urbana?

Lucrecia: Es un concepto... lo que pasa es que trayectorias... yo empecé pensando como en algo más estático como la gente que vive acá, qué significa vivir acá, cómo ven al barrio, cómo sentís que te ve el Estado, cuáles son los derechos, no sé.

Milena: Pero eso no es la idea de trayectoria.

Lucrecia: Bueno, pero empezó a surgir como que esa experiencia de vivir ahí está muy marcada también por experiencias anteriores habitacionales y, además, eso cruzado por la experiencia migratoria ¿no? como que me empecé a dar cuenta de que son cosas que se explican muy una a otra, digamos". (Fragmento observación taller de tesis II)

Como se observa, esta actividad resultaba formativa tanto para el alumno que expone su trabajo como para el que comenta, ya que tenían la posibilidad de observar otros modos de resolver la tarea (Boud y Lee, 2005). Asimismo, el intercambio con los otros atenuó la soledad usual del proceso de escritura (Carlino, 2012). Según Pasel y Asborno (1991) en el taller conviven tiempos para el trabajo grupal y tiempos para el trabajo individual. La cooperación entre los alumnos y el pensar juntos o co-pensar es constitutiva de esta estrategia. Así, el docente no ocupa un rol protagónico como poseedor del conocimiento sino que promueve el intercambio entre los doctorandos quienes, al analizar críticamente el trabajo de sus compañeros, favorecen el avance no solo de las tesis de sus pares sino también de la propia. Este intercambio tiene por resultado la formación en el quehacer de la investigación y también la socialización académica.

En último lugar, en los talleres de tesis observados esta estrategia de cooperación entre los alumnos se vio enriquecida por los variados niveles de expertise de los miembros del grupo. Allí convivían graduados recientes de la licenciatura con escaso recorrido en el ámbito académico con otros que contaban con una prolongada, aunque periférica, trayectoria en la comunidad académica. Los participantes más noveles suelen acudir a la orientación de sus pares más avanzados como un paso intermedio antes de consultar al docente. Lave (2011) también señala la importancia que allí tienen los intercambios entre pares donde uno de ellos se encuentra levemente por sobre el nivel de avance del otro.

En los párrafos anteriores mostramos cómo en los talleres los alumnos aprendían el repertorio de la comunidad, interactuaban con miembros de distinto nivel de participación y comenzaban a desarrollar tareas propias de la comunidad. Estas notas permiten pensar a los talleres como hitos de ingreso a la comunidad académica. El taller de tesis es una instancia dentro del programa doctoral, que es el hito de ingreso a la comunidad académica. La tesis es la primera tarea que enfrentan los investigadores en formación como miembros de la comunidad académica, siendo el proyecto y el plan los elementos que les permiten planificarla. Igualmente, es preciso señalar que la comunidad académica, en tanto comunidad de práctica, tiene un sistema más o menos restrictivo de participación para aquellos miembros que aún no transitaron el hito. Por ejemplo, en la Argentina solo alguien que ha obtenido el título de doctor puede acceder a la carrera de investigador del sistema científico nacional. Aun así, otros canales permanecen abiertos durante el proceso 
formativo como, por ejemplo, la publicación de artículos académicos o el acceso a algunos cargos de la gestión universitaria. Asimismo, es posible constatar que para algunos alumnos el taller no funciona como experiencia transicional de inserción en la comunidad sino más bien como una instancia legitimadora de su trayectoria en la academia.

\section{El taller: no siempre un espacio de enseñanza y aprendizaje}

Hasta aquí se expusieron los aspectos de los talleres observados que favorecen la aproximación de los alumnos a la práctica profesional. Sin embargo, tal como sostiene Perrenoud (2006), por momentos puede tornarse imposible sostener una posición genuina frente al aprendizaje durante la totalidad de una instancia formativa. Es decir, el alumno puede optar por posicionarse de manera estratégica frente al saber, y forjar una postura más ligada con la lógica de la aprobación que del aprendizaje (Brailovskyy Menchón, 2013). Ambas preocupaciones son legítimas y están presentes en toda situación de enseñanza. No obstante, desde la perspectiva de las comunidades de práctica se espera del alumno un deseo genuino de insertarse en la comunidad que exceda la mera aprobación del taller. Sin embargo, surgió de nuestras observaciones que lo opuesto ocurrió en uno de los talleres de tesis en el que algunos alumnos manifestaron que, presionados por el tipo de devoluciones recibidas y por las fechas de entrega, habían realizado un proyecto de tesis paralelo con el solo objeto de aprobar el taller pero conscientes de que esa no sería la investigación que llevarían a cabo.

Frecuentemente la omnipresencia de la lógica de la evaluación es la que promueve que el alumno se posicione como aprobante y ejerza su oficio de alumno (Perrenoud, 2006). Esto podía ocurrir por la cantidad de producciones que los doctorandos debían presentar, que favorecía el establecimiento de una relación estratégica con el taller en particular y con el conocimiento en general. Asimismo, en el caso del taller de tesis I el docente fomentaba en los alumnos la lógica de la aprobación al decirles que su objetivo era que su proyecto de tesis fuera aprobado por el programa doctoral, indistintamente de si este había sido ya aprobado por otra institución (como el sistema científico nacional). En este ejemplo, la lógica aprobante permea también la función del docente, quien se posiciona en un rol certificante.

Podríamos pensar que la lógica de la evaluación encuentra su punto máximo cuando los docentes se ubican en el rol de gatekeeper o guardián de la entrada a la comunidad, como ocurrió en algunos casos en los talleres observados. En el taller de tesis I durante los primeros encuentros el profesor indicó a una de las alumnas que su problema de tesis estaba mal construido, al punto de decirle, "No veo una tesis allí". El resultado fue que al cuarto encuentro la alumna dejó de asistir. Es decir, el profesor percibió que la estudiante no era capaz de formular un problema de investigación, una de las competencias básicas del investigador, y en lugar de orientar su aprendizaje le puso un límite a su inserción en la comunidad de práctica. Cabría pensar que el docente está en lo correcto pero el caso es que dicha alumna ya se encontraba trabajando con un director de tesis y contaba con una beca otorgada por el sistema científico nacional. Es decir, su proyecto de investigación ya había aprobado otras instancias evaluativas. Entonces, ¿cómo pueden ocurrir estas discrepancias? Demás está decir que distintas instituciones tienen distintos criterios de definición y aceptación de proyectos.

En definitiva, si bien el concepto de comunidad de práctica parece referir a relaciones armoniosas entre sus miembros, varios autores llaman la atención sobre la existencia de relaciones de poder que pueden favorecer u obstaculizar la inmersión de los nuevos miembros en la comunidad de práctica (Contu y Willmott, 2003; Hughes, Jewson y Unwin, 
2007; Owen-Pugh, 2007). Como se ha señalado en los párrafos anteriores, los alumnos pueden generar estrategias para enfrentar estas relaciones de poder, por ejemplo, cuando se posicionan en un rol aprobante. Mientras tanto, los docentes pueden ejercer su función de guardianes de la entrada a la comunidad.

\section{Conclusiones}

La pregunta que orientó este artículo fue, ¿Qué posibilidades brindan los talleres de tesis para pensar el aprendizaje de la práctica investigativa? Para responderla presentamos evidencias sobre el taller como puente entre la formación de posgrado y la práctica profesional (el quehacer de la investigación). La naturaleza de los saberes del oficio de la investigación requiere de dispositivos que acompañen y apuntalen su construcción en el propio proceso de hacer investigación. El taller parece ser central para el aprendizaje de estos saberes.

Dentro de estos saberes se encuentra la metodología de la investigación. En los talleres se sitúan y resignifican las nociones de metodología de la investigación y se ponen en juego contextualizadamente según cada tesis. Así, este artículo ilumina la discusión sobre la necesidad o no de transmisión de contenidos codificados de metodología de la investigación. Asimismo, son espacios centrados en el aprendizaje de la tarea, de los saberes del quehacer de la investigación que, para hacerlo, desarrollan estrategias de enseñanza centradas en el estudiante cuyo objetivo es guiar el proyecto y labor de cada uno: las guías de trabajo.

El artículo muestra, también, cómo el taller de tesis en el nivel de posgrado se presenta como un espacio pedagógico clave para la inserción en una comunidad de práctica: la comunidad académica. Los talleres observados también ponen en juego las tres características definitorias de una comunidad de práctica. Los talleres no solo parecen estar preparando a los estudiantes para insertarse en una práctica profesional sino que al mismo tiempo estarían funcionando como una micro-comunidad de práctica que replica la tarea, el repertorio y el compromiso mutuo de la comunidad de referencia. Estos tres elementos aparecen en el taller de manera más controlada y menos prolongada en el tiempo.

En el taller de tesis se desarrolla un espectro más o menos amplio de la tarea propia de la comunidad, pero esta tarea no se aborda en toda su complejidad. Por eso, podemos hablar de participación periférica: los nuevos se inician con tareas de menor compromiso y complejidad (Lave y Wenger, 1991). Aunque el foco del taller se encuentra en la tarea, en su desarrollo los alumnos adquieren el repertorio de la comunidad. Esto se ve favorecido porque, en ocasiones, la tarea a desempeñar en el marco del taller implica la inserción en las instituciones por las que circulan los miembros plenos de la comunidad. En el caso del compromiso, un punto a destacar es la relevancia otorgada en todos los talleres observados al intercambio entre pares y con el docente. En buena medida, la inserción en una comunidad se nutre de la participación y de la interacción con miembros de distinto grado de experiencia y conocimiento (expertise).

En definitiva, los autores consideran que el marco de comunidades de práctica es propicio para el abordaje de la transmisión de saberes prácticos en el marco de la educación de 
posgrado. Se trata de una perspectiva que, aunque no se enfoca en lo que sucede en la institución escolar, toma al aprendizaje como un elemento constitutivo en la conformación de una comunidad de práctica. Por ello resulta relevante para el análisis de dispositivos pedagógicos como los talleres en los que la tarea gira en torno a prácticas concretas. Este artículo aporta a la definición y caracterización pedagógico-didáctica de los talleres de tesis. Todavía es necesario continuar con esta línea de investigación para profundizar nuestro conocimiento sobre los saberes que se transmiten y las estrategias didácticas que se ponen en juego en los talleres de tesis de posgrado.

\section{Referencias bibliográficas}

Arnoux, E. Narvaja de, Borsinger, A., Carlino, P., di Stefano, M., Pereira, C. y Silvestre, A. (2004). La intervención pedagógica en el proceso de escritura de tesis de posgrado. Revista de la Maestría en Salud Pública, 3(6). https://www.aacademica.org/paula.carlino/169.pdf

Atkinson, T. y Claxton, G. (2000). The intuitive practitioner: On the value of not always knowing what one is doing. Buckingham: Open University Press.

Barab, S., Barnett, M. y Squire, K. (2002). Developing an empirical account of a community of practice: Characterizing the essential tensions. Journal of the Learning Sciences, 11(4), 489-542.

Barros, N. A. de (1977). El taller, integración de teoría y práctica. Buenos Aires: Editorial Humanitas.

Baruque, J. V. (2001). Gremios y oficios en la estructura urbana de la ciudad medieval. En A. Ciudad Ruiz, M. J. Iglesias Ponce de León y Ma. del C. Martínez Martínez (comps.), Reconstruyendo la ciudad maya: el urbanismo en las sociedades antiguas (pp. 483-490). Valladolid: Sociedad Española de Estudios Mayas. https://dialnet.unirioja.es/servlet/ articulo?codigo $=2776132$

Boud, D. y Lee, A. (2005). 'Peer learning' as pedagogic discourse for research education. Studies in Higher Education, 30(5), 501-516. doi: 10.1080/03075070500249138 https:// www.researchgate.net/publication/232826954_\%27Peer learning\%27_as_pedagogic discourse for research education

Bourdieu, P., Chamboredon, J.-C. y Passeron, J.-C. (2008). El oficio de sociólogo. Presupuestos epistemológicos. Argentina: Siglo XXI Editores.

Brailovsky, D. y Menchón, Á. (2013). Estrategias de escritura en la formación. Buenos Aires: Noveduc.

Buysse, V., Sparkman, K. y Wesley, P. (2003). Communities of practice: Connecting what we know with what we do. Exceptional Children, 69(3), 263-277. doi: 10.1177/001440290306900301

Carlino, P. (2012). Helping doctoral students of education to face writing and emotional challenges in identity transition. En M. Castelló y C. Donahue (comps.), University writing: Selves and texts in academic societies (pp. 217-234). London: Emeral Group. 
Carrasco Altamirano, A. y Kent Serna, R. (2011). Leer y escribir en el doctorado o el reto de formarse como autor de ciencias. Revista mexicana de investigación educativa, 16(51), 1227-1251. http://www.scielo.org.mx/pdf/rmie/v16n51/v16n51a10.pdf

Clark, B. (1995 [1989]). Places of inquiry. Research and Advanced Education in Modern Universities. Berkeley: University of California Press.

Contu, A. y Willmott, H. (2003). Re-embedding situatedness: The importance of power relations in learning theory. Organization Science, 14(3), 283-296. http://citeseerx.ist.psu. edu/viewdoc/download?doi=10.1.1.203.3070\&rep=rep1\&type=pdf

De Vita, A. (2012). La creación social: relaciones y contextos para educar. Barcelona: Laertes.

Difabio, H. y Heredia, M. del V. (2013). El taller de tesis doctoral en educación desde un enfoque comprehensivo de escritura a través de la plataforma Moodle. Presentado en VI Seminario Internacional de Educación a Distancia, Argentina. http://www.uncu.edu.ar/ seminario_rueda/upload/t234.pdf

Eckelberry, R. H. (1940). The voluntary doctoral seminar. The Journal of Higher Education, 11(7), 367-370.

Faiver, C. M., McNally, C. J. y Nims, P. J. (2000). Teaching a workshop on creativity and intuition in counseling. Journal of Humanistic Counseling, Education and Development, 38(4), 220-229. doi: 10.1002/j.2164-490X.2000.tb00083.x

Fuller, A. (2007). Critiquing theories of learning and communities of practice. En J. Hughes, N. Jewson y L. Unwin (comps.), Communities of practice: Critical perspectives. London: Routledge.

Goodwin, J. (2007). The transition to work and adulthood. En J. Hughes, N. Jewson y L. Unwin (comps.), Communities of practice: Critical perspectives. London: Routledge.

Hughes, J., Jewson, N. y Unwin, L. (2007). Communities of practice: Critical perspectives. London: Routledge.

Jones, S. (2000). 'Enlivening' development concepts through workshops: A case study of appropriate technology and soil conservation. Journal of Geography in Higher Education, 24(1), 75-86. doi: 10.1080/03098260085144

Kiley, M. (1996). How do I know how I am going? Assessment in post graduate research degrees. En Quality in postgraduate research: Is it happening? Adelaide: University of Adelaide.

Lave, J. (2011). Apprenticeship in critical ethnographic practice. Chicago: University of Chicago Press.

Lave, J. y Wenger, E. (1991). Situated learning: Legitimate peripheral participation. Cambridge: Cambridge University Press.

Litwin, E. (2008). El oficio de enseñar. Condiciones y contextos. Buenos Aires: Paidós. 
Lopes Espinoza, S. y Weiss, E. (2007). Una mirada diferente a las prácticas: un taller de electrónica en el CONALEP. Revista Mexicana de Investigación Educativa, 12(35), 1329-1356.

Lovitts, B. (2005). Being a good course-taker is not enough: a theoretical perspective on the transition to independent research. Studies in Higher Education, 30(2), 137-154.

Menjot, D. (2010). El mundo del artesanado y la industria en las ciudades de Europa occidental durante la Edad Media (siglos XII-XV). CATHARUM Revista de Ciencias y Humanidades del Instituto de Estudios Hispánicos de Canarias, 11(3), 5-18.

Narvaja de Arnoux, E. (Dir.) (2009) Funciones de la escritura en el capítulo de tesis y en la defensa oral de la tesis. En E. Narvaja de Arnoux, (2009) Escritura y producción de conocimiento en las carreras de posgrado (pp. 143-168). Buenos Aires: Santiago Arcos editor. http://www.scielo.cl/scielo.php?script=sci_arttext\&pid=S0718-09342013000300007

Owen-Pugh, V. (2007). Theorizing sport as a community of practice. The coach-athlete relationship in British professional basketball. En N. Jewson, J. Hughes y L. Unwin (comps.), Communities of practice: Critical perspectives. London: Routledge.

Pasel, S. y Asborno, S. (1991). Aula-taller. Buenos Aires: Aique.

Perrenoud, P. (2006). El oficio de alumno y el sentido del trabajo escolar. Madrid: Popular.

Perry, J. (2004). Authentic learning in field schools: preparing future members of the archaeological community. World Archaeology, 36(2), 236-260.

Schön, D. (1992). La formación de profesionales reflexivos: hacia un nuevo diseño de la enseñanza y el aprendizaje en las profesiones. Barcelona: Paidós.

Sennett, R. (2009). El artesano. Barcelona: Anagrama.

Vitale, M. A. (2009). La reformulación de un proyecto de tesis de posgrado y el proceso de aprendizaje de un campo disciplinario. En E. Narvaja de Arnoux, Escritura y producción de conocimiento en las carreras de posgrado (pp. 121-137). Buenos Aires: Santiago Arcos editor.

Wainerman, C. (2011). Acerca de la formación de investigadores en ciencias sociales. En C. Wainerman y R. Sautu (Eds.), La trastienda de la investigación (pp. 19-26). Buenos Aires: Manantial. http://www.fapyd.unr.edu.ar/wp-content/uploads/2015/09/WainermanSautu-indice-cap-1.pdf

Wenger, E. (1998). Communities of practice: learning, meaning, and identity. Cambridge: Cambridge University Press.

Wright Mills, C. (1959). The sociological imagination. New York: Oxford University Press. 


\section{(Endnotes)}

${ }^{1}$ Esta investigación fue realizada gracias a las becas doctorales otorgadas por el Consejo Nacional de Investigaciones Científicas y Tecnológicas a cada una de las autoras.

2 Espacios de larga data, el primero del que tenemos referencia es de 1940 (Eckelberry, 1940), y existentes en variadas disciplinas, de carácter informal y optativo a los que los doctorandos asisten para discutir avances de la tesis.

${ }^{3}$ En la estructura curricular de un programa doctoral los talleres de tesis podrían ser instancias institucionales que complementan el trabajo con el director de tesis. Aunque el vínculo entre ambos excede los fines de este trabajo, sería interesante analizar la relación entre ambas instancias formativas en un futuro estudio.

${ }^{4}$ Aquí se incluirán las referencias a la tesis doctoral y a su director.

${ }^{5}$ El proyecto de investigación comprende los siguientes elementos: título, área disciplinar, resumen, objetivos generales y específicos, estado de la cuestión, metodología, actividades, lugar de trabajo y factibilidad y bibliografía.

${ }^{6}$ El plan de tesis debe presentarse luego de haber recolectado los datos para la investigación y comprende los siguientes elementos: título de la tesis, director/a de trabajo de tesis, resumen de la tesis, síntesis de la pregunta-problema, modo de abordaje y resultados, índice analítico y comentado de la tesis y bibliografía.

${ }^{7}$ Se utilizaron seudónimos con el fin de resguardar la identidad de los participantes. Asimismo, se omitieron los datos de las investigaciones que pudieran resultar en su identificación. Los docentes son: Bernardo, Mónica y Mauro. 